PROCEEDINGS OF THE

AMERICAN MATHEMATICAL SOCIETY

Volume 127, Number 7, Pages 2065-2071

S 0002-9939(99)04798-X

Article electronically published on February 26, 1999

\title{
THE WIENER TRANSFORM ON THE BESICOVITCH SPACES
}

\author{
CHRISTOPHER HEIL
}

(Communicated by J. Marshall Ash)

\begin{abstract}
In his fundamental research on generalized harmonic analysis, Wiener proved that the integrated Fourier transform defined by $W f(\gamma)=$ $\int f(t)\left(e^{-2 \pi i \gamma t}-\chi_{[-1,1]}(t)\right) /(-2 \pi i t) d t$ is an isometry from a nonlinear space of functions of bounded average quadratic power into a nonlinear space of functions of bounded quadratic variation. We consider this Wiener transform on the larger, linear, Besicovitch spaces $\mathcal{B}_{p, q}(\mathbf{R})$ defined by the norm $\|f\|_{\mathcal{B}_{p, q}}=\left(\int_{0}^{\infty}\left(\frac{1}{2 T} \int_{-T}^{T}|f(t)|^{p} d t\right)^{q / p} \frac{d T}{T}\right)^{1 / q}$. We prove that $W$ maps $\mathcal{B}_{p, q}(\mathbf{R})$ continuously into the homogeneous Besov space $\dot{B}_{p^{\prime}, q}^{1 / p^{\prime}}(\mathbf{R})$ for $1<p \leq 2$ and $1<q \leq \infty$, and is a topological isomorphism when $p=2$.
\end{abstract}

\section{INTRODUCTION}

In his fundamental research on generalized harmonic analysis, Wiener [W] proved that the integrated Fourier transform, or Wiener transform,

$$
W f(\gamma)=\int_{-\infty}^{\infty} f(t) \frac{e^{-2 \pi i \gamma t}-\chi_{[-1,1]}(t)}{-2 \pi i t} d t
$$

satisfies the isometry relation

$$
\lim _{T \rightarrow \infty} \frac{1}{2 T} \int_{-T}^{T}|f(t)|^{2} d t=\lim _{\lambda \rightarrow 0} \frac{2}{\lambda} \int_{-\infty}^{\infty}\left|\Delta_{\lambda} W f(\gamma)\right|^{2} d \gamma
$$

where $\Delta_{\lambda}$ is the symmetric difference operator $\Delta_{\lambda} F(\gamma)=F(\gamma+\lambda)-F(\gamma-\lambda)$. Equation (1) is the Wiener-Plancherel formula.

The space of functions for which the limit of the quadratic averages on the lefthand side of (1) exists is nonlinear [HW], and therefore cannot be dealt with using the methods of ordinary functional analysis. It is therefore natural to consider the Wiener transform on larger linear spaces. The Marcinkiewicz space $M^{p}(\mathbf{R})$ is defined by the norm $\|f\|_{M^{p}}=\lim \sup _{T \rightarrow \infty}\left(\frac{1}{2 T} \int_{-T}^{T}|f(t)|^{p} d t\right)^{1 / p}$. Marcinkiewicz himself referred to this space as a Besicovitch space $[\mathrm{M}]$. The usual Besicovitch space formed by completing the space of almost periodic functions is closely related.

Received by the editors August 20, 1996 and, in revised form, October 8, 1997.

1991 Mathematics Subject Classification. Primary 42A38; Secondary 42A75, 46B03.

Key words and phrases. Besicovitch spaces, Besov spaces, Marcinkiewicz spaces, WienerPlancherel formula, Wiener transform.

This research was supported by National Science Foundation Grant DMS-9401340.

(C)1999 American Mathematical Society 
We shall deal with another collection of closely related spaces $\mathcal{B}_{p, q}(\mathbf{R})$, defined by the norm

$$
\|f\|_{\mathcal{B}_{p, q}}= \begin{cases}\left(\int_{0}^{\infty}\left(\frac{1}{2 T} \int_{-T}^{T}|f(t)|^{p} d t\right)^{q / p} \frac{d T}{T}\right)^{1 / q}, & q<\infty \\ \sup _{T>0}\left(\frac{1}{2 T} \int_{-T}^{T}|f(t)|^{p} d t\right)^{1 / p}, & q=\infty .\end{cases}
$$

By extension, we refer to $\mathcal{B}_{p, q}(\mathbf{R})$ as a Besicovitch space.

Chen and Lau [CL] considered the Wiener transform on $\mathcal{B}_{2, \infty}(\mathbf{R})$, and proved that $W$ is a topological isomorphism of $\mathcal{B}_{2, \infty}(\mathbf{R})$ onto a space $V$ defined by the norm $\|F\|_{V}=\sup _{\lambda>0}\left(\frac{2}{\lambda} \int_{-\infty}^{\infty}\left|\Delta_{\lambda} F(\gamma)\right|^{2} d \gamma\right)^{1 / 2}$. This space $V$ coincides with the homogeneous Besov space $\dot{B}_{2, \infty}^{1 / 2}(\mathbf{R})$. It is our purpose in this paper to consider the Wiener transform on the Besicovitch space $\mathcal{B}_{p, q}(\mathbf{R})$ for $1<p \leq 2,1<q \leq \infty$, and to connect this study to Beurling's fundamental work on spectral synthesis [Beur].

\section{Notation}

Feichtinger $[\mathrm{F}]$ has observed that the Besicovitch space $\mathcal{B}_{p, q}(\mathbf{R})$ coincides (in the sense of equivalent norms) with the "dyadic" amalgam space $\left(L^{p}\left(\mathbf{R}^{\times}\right), \ell^{q}\right)$, where $L^{p}\left(\mathbf{R}^{\times}\right)$is the Lebesgue space with respect to the Haar measure $d t /|t|$ on the multiplicative group $\mathbf{R}^{\times}$of nonzero real numbers. This amalgam space is defined by the norm

$$
\|f\|_{\left(L^{p}\left(\mathbf{R}^{\times}\right), \ell q\right)}=\left(\sum_{n \in \mathbf{Z}}\left(\int_{2^{n} \leq|t| \leq 2^{n+1}}|f(t)|^{p} \frac{d t}{|t|}\right)^{q / p}\right)^{1 / q} .
$$

As a consequence, $\mathcal{B}_{p, q}(\mathbf{R})$ is a Banach space and, for $1 \leq p, q<\infty$, its dual is $\left(\mathcal{B}_{p, q}(\mathbf{R})\right)^{\prime}=\mathcal{B}_{p^{\prime}, q^{\prime}}(\mathbf{R})$ when the duality is defined by

$$
\langle f, g\rangle=\int_{0}^{\infty}\left(\frac{1}{2 T} \int_{-T}^{T} f(t) \overline{g(t)} d t\right) \frac{d T}{T}=\frac{1}{2} \int f(t) \overline{g(t)} \frac{d t}{|t|} .
$$

As usual, $\frac{1}{p}+\frac{1}{p^{\prime}}=\frac{1}{q}+\frac{1}{q^{\prime}}=1$.

It is a standard fact $[\mathrm{T}]$ that, for the range of indices $1 \leq p, q<\infty$, and $0<s<1$, an equivalent norm for the homogeneous Besov space $\dot{B}_{p, q}^{s}(\mathbf{R})$ is given by

$$
\|F\|_{\dot{B}_{p, q}^{s}}=\left(\int_{0}^{\infty}\left(\frac{2}{\lambda^{s}}\left(\int_{-\infty}^{\infty}\left|\Delta_{\lambda} F(\gamma)\right|^{p} d \gamma\right)^{1 / p}\right)^{q} \frac{d \lambda}{\lambda}\right)^{1 / q}
$$

The usual adjustment is made when $q=\infty$.

The absolute value of the Dirichlet kernel will play an important role in our results. We use the notation

$$
S(t)=\left|\frac{\sin 2 \pi t}{\pi t}\right| \quad \text { and } \quad S_{\lambda}(t)=\lambda S(\lambda t)=\left|\frac{\sin 2 \pi \lambda t}{\pi t}\right| .
$$

Given an even, nonnegative function $w$ on $\mathbf{R}$, we define its greatest decreasing minorant $w_{*}$ and least decreasing majorant $w^{*}$ by

$$
w_{*}(t)=\inf _{0 \leq|u| \leq|t|} w(u) \quad \text { and } \quad w^{*}(t)=\sup _{|t| \leq|u|} w(u) .
$$


Note that $w_{*}(t) \leq w(t) \leq w^{*}(t)$ for all $t$, and that $w_{*}, w^{*}$ are nonnegative, even functions that are decreasing on $[0, \infty)$. Also note in particular that $S^{*} \in L^{p}(\mathbf{R})$ if $p>1$, and that $S_{*}=S \cdot \chi_{[-\pi / 4, \pi / 4]}$.

The Fourier transform is $\hat{f}(\gamma)=\int f(t) e^{-2 \pi i \gamma t} d t$, and the inverse Fourier transform is $\check{f}(\gamma)=\int f(t) e^{2 \pi i \gamma t} d t$.

\section{The Wiener transform ON $\mathcal{B}_{p, q}(\mathbf{R})$}

We shall prove that the Wiener transform is a topological isomorphism of the Besicovitch space $\mathcal{B}_{2, q}(\mathbf{R})$ onto the homogeneous Besov space $\dot{B}_{2, q}^{1 / 2}(\mathbf{R})$ for $1<$ $q \leq \infty$, and that $W$ maps $\mathcal{B}_{p, q}(\mathbf{R})$ continuously into $\dot{B}_{p^{\prime}, q}^{1 / p^{\prime}}(\mathbf{R})$ for $1<p \leq 2$ and $1<q \leq \infty$.

The following fact will be useful: if $w$ is an even, nonnegative function on $\mathbf{R}$ that is decreasing on $[0, \infty)$, then for each $\alpha>1$ we have

$$
\sum_{n \in \mathbf{Z}} \alpha^{n-1} w\left(\alpha^{n}\right) \leq \frac{1}{\alpha-1} \int_{0}^{\infty} w(t) d t \leq \sum_{n \in \mathbf{Z}} \alpha^{n} w\left(\alpha^{n}\right) .
$$

The following lemma establishes some basic facts about the Wiener transform.

Lemma 1. Fix $1<p \leq 2$ and $1 \leq q \leq \infty$. If $f \in \mathcal{B}_{p, q}(\mathbf{R})$, then the following statements hold for each $\lambda>0$.

(a) $f \cdot S_{\lambda} \in L^{p}(\mathbf{R})$.

(b) $\Delta_{\lambda}(W f)=\frac{1}{2}\left(f \cdot S_{\lambda}\right)^{\wedge} \in L^{p^{\prime}}(\mathbf{R})$.

(c) $\|W f\|_{\dot{B}_{p^{\prime}, q}^{1 / p^{\prime}}} \leq\left(\int_{0}^{\infty}\left(\frac{\lambda}{2} \int_{-\infty}^{\infty}|f(t)|^{p} S(\lambda t)^{p} d t\right)^{q / p} \frac{d \lambda}{\lambda}\right)^{1 / q}$, with equality if $p=2$.

Proof. (a) Set $I_{n}=\left[-2^{n+1},-2^{n}\right] \cup\left[2^{n}, 2^{n+1}\right]$. Then for each $\lambda>0$,

$$
\begin{aligned}
\int_{-\infty}^{\infty}\left|f(t) S_{\lambda}(t)\right|^{p} d t & \leq \lambda^{p} \sum_{n \in \mathbf{Z}} \int_{I_{n}}|f(t)|^{p} S^{*}(\lambda t)^{p} \frac{2^{n+1}}{|t|} d t \\
& \leq \lambda^{p}\left(\sum_{n \in \mathbf{Z}} 2^{n+1} S^{*}\left(2^{n} \lambda\right)^{p}\right)\left(\sup _{n \in \mathbf{Z}} \int_{I_{n}}|f(t)|^{p} \frac{d t}{|t|}\right) \\
& \leq 4 \lambda^{p}\left(\int_{0}^{\infty} S^{*}(\lambda t)^{p} d t\right)\|f\|_{\left(L^{p}\left(\mathbf{R}^{\times}\right), \ell^{\infty}\right)},
\end{aligned}
$$

the last inequality following from (2). Since $S^{*} \in L^{p}(\mathbf{R})$ for $p>1$ and since $\mathcal{B}_{p, q}(\mathbf{R})=\left(L^{p}\left(\mathbf{R}^{\times}\right), \ell^{q}\right) \subset\left(L^{p}\left(\mathbf{R}^{\times}\right), \ell^{\infty}\right)$, the result follows.

(b) Follows from a direct computation and from part (a).

(c) By the Hausdorff-Young inequality,

$$
\begin{aligned}
\|W f\|_{\dot{B}_{p^{\prime}, q}^{1 / p^{\prime}}} & =\left(\int_{0}^{\infty}\left(\frac{2}{\lambda} \int_{-\infty}^{\infty}\left|\Delta_{\lambda} W f(\gamma)\right|^{p^{\prime}} d \gamma\right)^{q / p^{\prime}} \frac{d \lambda}{\lambda}\right)^{1 / q} \\
& \leq\left(\int_{0}^{\infty}\left(\frac{2}{\lambda}\right)^{q / p^{\prime}}\left(\int_{-\infty}^{\infty}\left|\frac{1}{2} f(t) S_{\lambda}(t)\right|^{p} d t\right)^{q / p} \frac{d \lambda}{\lambda}\right)^{1 / q} \\
& =\left(\int_{0}^{\infty}\left(\frac{\lambda}{2} \int_{-\infty}^{\infty}|f(t)|^{p} S(\lambda t)^{p} d t\right)^{q / p} \frac{d \lambda}{\lambda}\right)^{1 / q}
\end{aligned}
$$

with equality if $p=2$. 
The following two results estimate integrals of the form in Lemma 1(c).

Proposition 2. Let $w$ be even and nonnegative on $\mathbf{R}$, and fix $1 \leq p<\infty$ and $1 \leq q \leq \infty$. Then

$$
\left(\int_{0}^{\infty}\left(\frac{\lambda}{2} \int_{-\infty}^{\infty}|f(t)|^{p} w(\lambda t) d t\right)^{q / p} \frac{d \lambda}{\lambda}\right)^{1 / q} \leq C\|f\|_{\mathcal{B}_{p, q}}
$$

with

$$
C= \begin{cases}\left(\int_{0}^{\infty} w^{*}(t) d t\right)^{1 / p}, & \text { if } p \leq q, \\ 4^{1 / q}\left(\sup _{0 \leq t \leq 1} w^{*}(t)^{q / p}+\int_{1}^{\infty} w^{*}(t)^{q / p} d t\right)^{1 / q}, & \text { if } q<p .\end{cases}
$$

Proof. Fix $\alpha>1$, and define $\omega_{n}=w^{*}\left(\alpha^{n}\right)-w^{*}\left(\alpha^{n+1}\right)$. Then, since $w^{*}$ is even and is decreasing on $[0, \infty)$,

$$
\sum_{n \in \mathbf{Z}} \omega_{n} \chi_{\left[-\alpha^{n}, \alpha^{n}\right]}(t) \leq w^{*}(t) \leq \sum_{n \in \mathbf{Z}} \omega_{n} \chi_{\left[-\alpha^{n+1}, \alpha^{n+1}\right]}(t) .
$$

Assume now that $1 \leq p \leq q<\infty$ (the case $q=\infty$ is similar). Then, using (3), the change of variables $T=\alpha^{n+1} / \lambda$, and the triangle inequality in the Banach space $L^{q / p}\left(\mathbf{R}^{\times}\right)$, we compute

$$
\begin{aligned}
\left(\int_{0}^{\infty}\right. & \left.\left(\frac{\lambda}{2} \int_{-\infty}^{\infty}|f(t)|^{p} w(\lambda t) d t\right)^{q / p} \frac{d \lambda}{\lambda}\right)^{p / q} \\
& \leq\left(\int_{0}^{\infty}\left(\frac{\lambda}{2} \sum_{n \in \mathbf{Z}} \omega_{n} \int_{-\alpha^{n+1} / \lambda}^{\alpha^{n+1} / \lambda}|f(t)|^{p} d t\right)^{q / p} \frac{d \lambda}{\lambda}\right)^{p / q} \\
& =\left(\int_{0}^{\infty}\left(\sum_{n \in \mathbf{Z}} \frac{\alpha^{n+1} \omega_{n}}{2 T} \int_{-T}^{T}|f(t)|^{p} d t\right)^{q / p} \frac{d T}{T}\right)^{p / q} \\
& \leq\left(\sum_{n \in \mathbf{Z}} \alpha^{n+1} \omega_{n}\right)\|f\|_{\mathcal{B}_{p, q}}^{p} \\
& \leq\left(\alpha \int_{0}^{\infty} w^{*}(t) d t\right)\|f\|_{\mathcal{B}_{p, q}^{p}}^{p}
\end{aligned}
$$

the last inequality following by integrating (3) over [0, ). Letting $\alpha \rightarrow 1$ therefore gives the result.

For the case $1 \leq q<p<\infty$, we use instead the triangle inequality in the metric space $L^{q / p}\left(\mathbf{R}^{\times}\right)$to derive the inequality

$$
\int_{0}^{\infty}\left(\sum_{n \in \mathbf{Z}} \frac{\alpha^{n+1} \omega_{n}}{2 T} \int_{-T}^{T}|f(t)|^{p} d t\right)^{q / p} \frac{d T}{T} \leq \sum_{n \in \mathbf{Z}}\left(\alpha^{n+1} \omega_{n}\right)^{q / p}\|f\|_{\mathcal{B}_{p, q}}^{q} .
$$

Then, taking $\alpha=2^{p / q}$, we have the estimates

$$
\begin{aligned}
& \sum_{n>0}\left(\alpha^{n+1} \omega_{n}\right)^{q / p} \leq \sum_{n>0} 2^{n+1} w^{*}\left(2^{n}\right)^{q / p} \leq 4 \int_{1}^{\infty} w^{*}(t)^{q / p} d t \\
& \sum_{n \leq 0}\left(\alpha^{n+1} \omega_{n}\right)^{q / p} \leq \sum_{n \leq 0} 2^{n+1} \sup _{0 \leq t \leq 1} w^{*}(t)^{q / p} \leq 4 \sup _{0 \leq t \leq 1} w^{*}(t)^{q / p} .
\end{aligned}
$$


Proposition 3. Let $w$ be even and nonnegative on $\mathbf{R}$, and fix $1 \leq p, q \leq \infty$. Then

$$
\left(\int_{0}^{\infty}\left(\frac{\lambda}{2} \int_{-\infty}^{\infty}|f(t)|^{p} w(\lambda t) d t\right)^{q / p} \frac{d \lambda}{\lambda}\right)^{1 / q} \geq\left(\sup _{b \in \mathbf{R}} b w_{*}(b)\right)^{1 / p}\|f\|_{\mathcal{B}_{p, q}} .
$$

Proof. Assume that $1 \leq p, q<\infty$ (the cases $p=\infty$ or $q=\infty$ are similar). Fix $b \in \mathbf{R}$. Then, using the change of variables $\lambda=b / T$, we compute

$$
\begin{aligned}
\int_{0}^{\infty}\left(\frac{\lambda}{2} \int_{-\infty}^{\infty}|f(t)|^{p} w(\lambda t) d t\right)^{q / p} \frac{d \lambda}{\lambda} & \geq \int_{0}^{\infty}\left(\frac{b}{2 T} \int_{-\infty}^{\infty}|f(t)|^{p} w_{*}(b t / T) d t\right)^{q / p} \frac{d T}{T} \\
& \geq \int_{0}^{\infty}\left(\frac{b}{2 T} \int_{-T}^{T}|f(t)|^{p} w_{*}(b) d t\right)^{q / p} \frac{d T}{T} \\
& =\left(b w_{*}(b)\right)^{q / p}\|f\|_{\mathcal{B}_{p, q}}^{q}
\end{aligned}
$$

Theorem 4. (a) If $1<p \leq 2$ and $1<q \leq \infty$, then $W$ maps $\mathcal{B}_{p, q}(\mathbf{R})$ continuously into $\dot{B}_{p^{\prime}, q}^{1 / p^{\prime}}(\mathbf{R})$.

(b) $W$ is a topological isomorphism of $\mathcal{B}_{2, q}(\mathbf{R})$ onto $\dot{B}_{2, q}^{1 / 2}(\mathbf{R})$ for $1<q \leq \infty$.

Proof. (a) In light of Lemma 1, apply Proposition 2 using $w(t)=S(t)^{p}$. The result follows for $p \leq q \leq \infty$ since $\left(S^{*}\right)^{p}$ is integrable, and for $1<q \leq p$ since $\left(S^{*}\right)^{p \cdot q / p}=\left(S^{*}\right)^{q}$ is integrable and bounded.

(b) Since $0<\sup \left(b S_{*}(b)^{2}\right)<\infty$, it follows from Proposition 3 that $W^{-1}$ : Range $(\mathrm{W}) \rightarrow \mathcal{B}_{2, q}(\mathbf{R})$ is continuous. It therefore remains only to show that $W$ is surjective. Fix $G \in \dot{B}_{2, q}^{1 / 2}(\mathbf{R})$. Then $\Delta_{\lambda} G \in L^{2}(\mathbf{R})$ for almost every $\lambda$. Set $\beta_{\lambda}(t)=-i \sin 2 \pi \lambda t$, and define $f_{\lambda}=\left(\Delta_{\lambda} G\right)^{\vee} / \beta_{\lambda}$. Then $\beta_{\mu} \beta_{\lambda} f_{\lambda}=\left(\Delta_{\mu} \Delta_{\lambda} G\right)^{\vee}=$ $\left(\Delta_{\lambda} \Delta_{\mu} G\right)^{\vee}=\beta_{\lambda} \beta_{\mu} f_{\mu}$ and $\beta_{\lambda} \beta_{\mu}$ is nonzero a.e., so $f=f_{\lambda}$ is independent of $\lambda$. Define $g(t)=-2 \pi i t f(t)$. Then, since $\left(\Delta_{\lambda} G\right)^{\vee}(t)=-i(\sin 2 \pi \lambda t) f(t)=\frac{\lambda}{2} S(\lambda t) g(t)$, we have by the Plancherel formula and Proposition 3 that

$$
\begin{aligned}
\|G\|_{\dot{B}_{2, q}^{1 / 2}}^{q} & =\int_{0}^{\infty}\left(\frac{2}{\lambda} \int_{-\infty}^{\infty}\left|\Delta_{\lambda} G(\gamma)\right|^{2} d \gamma\right)^{q / 2} \frac{d \lambda}{\lambda} \\
& =\int_{0}^{\infty}\left(\frac{\lambda}{2} \int_{-\infty}^{\infty}|g(t)|^{2} S(\lambda t)^{2} d t\right)^{q / 2} \frac{d \lambda}{\lambda} \geq C\|g\|_{\mathcal{B}_{2, q}}^{q} .
\end{aligned}
$$

Hence $g \in \mathcal{B}_{2, q}(\mathbf{R})$, and therefore $W g \in \dot{B}_{2, q}^{1 / 2}(\mathbf{R})$. Finally, $\Delta_{\lambda} W g=\frac{1}{2}\left(g \cdot S_{\lambda}\right)^{\wedge}=$ $\left(\beta_{\lambda} \cdot f\right)^{\wedge}=\Delta_{\lambda} G$, so $W g=G$ in $\dot{B}_{2, q}^{1 / 2}(\mathbf{R})$.

\section{Connection to Beurling's $A^{p}, B^{p}$ spaces}

In one of his deep investigations into spectral synthesis, Beurling [Beur] introduced the spaces

$$
A^{p}=\bigcup_{w \in \Omega} L_{w^{1-p}}^{p}(\mathbf{R}) \quad \text { and } \quad B^{p}=\bigcap_{w \in \Omega} L_{w}^{p}(\mathbf{R}) .
$$

Here $\Omega$ is the class of all even, positive, integrable functions on $\mathbf{R}$, and the weighted $L^{p}$ space $L_{w}^{p}(\mathbf{R})$ is defined by the norm $\|f\|_{L_{w}^{p}}=\left(\int|f(t)|^{p} w(t) d t\right)^{1 / p}$. $A^{p}$ is a convolution algebra contained in $L^{1}(\mathbf{R})$. The spaces $A^{p}$ and $B^{p^{\prime}}$ are duals when 
the duality is defined by $\langle f, g\rangle=\int f(t) \overline{g(t)} d t$. Moreover, $B^{p}$ coincides with the Besicovitch space $\mathcal{B}_{p, \infty}(\mathbf{R})$, with equality of norms [Beur].

By using clever estimates on the weights $w$, Beurling proved that the Fourier transform is a topological isomorphism of $A^{2}$ onto a space $U$ defined by the norm $\|F\|_{U}=\int_{0}^{\infty}\left(\frac{2}{\lambda} \int_{-\infty}^{\infty}\left|\Delta_{\lambda} F(\gamma)\right|^{2} d \gamma\right)^{1 / 2} \frac{d \lambda}{\lambda}$. This space $U$ is the homogeneous Besov space $\dot{B}_{2,1}^{1 / 2}(\mathbf{R})$. We will show in this section how Beurling's isomorphism result for the Fourier transform connects to our isomorphism result for the Wiener transform.

The following lemma establishes the relationship between $A^{p}$ and $\mathcal{B}_{p, 1}(\mathbf{R})$.

Lemma 5. $A^{p}=\left\{t f(t): f \in \mathcal{B}_{p, 1}(\mathbf{R})\right\}$, with $\|f\|_{A^{p}}=2\|t f(t)\|_{\mathcal{B}_{p, 1}}$.

Proof. This is a consequence of the following facts:

(a) $\left(A^{p}\right)^{\prime}=B^{p^{\prime}}$ under the duality $\langle f, g\rangle=\int f(t) \overline{g(t)} d t$,

(b) $\left(\mathcal{B}_{p, 1}(\mathbf{R})\right)^{\prime}=\mathcal{B}_{p^{\prime}, \infty}(\mathbf{R})$ under the duality $\langle f, g\rangle=\frac{1}{2} \int f(t) \overline{g(t)} \frac{d t}{|t|}$,

(c) $B^{p^{\prime}}=\mathcal{B}_{p^{\prime}, \infty}(\mathbf{R})$, with equality of norms.

Theorem 6. $W$ is a topological isomorphism of $\mathcal{B}_{2,1}(\mathbf{R})$ onto $\dot{B}_{2,1}^{1 / 2}(\mathbf{R})$.

Proof. The fact that the Fourier transform is a topological isomorphism of $A^{2}$ onto $\dot{B}_{2,1}^{1 / 2}(\mathbf{R})$ implies that $C_{1}\|g\|_{A^{2}} \leq\|\hat{g}\|_{\dot{B}_{2,1}^{1 / 2}} \leq C_{2}\|g\|_{A^{2}}$ for all $g \in A^{2}$.

Fix now any $f \in \mathcal{B}_{2,1}(\mathbf{R})$. Then, by Lemma $5, g(t)=f(t) / t \in A^{2} \subset L^{1}(\mathbf{R})$, with $\|g\|_{A^{2}}=2\|f\|_{\mathcal{B}_{2,1}}$. Since $f(t) / t$ is integrable, we have that

$$
\Delta_{\lambda} W f(\gamma)=\int_{-\infty}^{\infty} \frac{f(t)}{-2 \pi i t}\left(e^{-2 \pi i(\gamma+\lambda) t}-e^{-2 \pi i(\gamma-\lambda) t}\right) d t=-\frac{1}{2 \pi i} \Delta_{\lambda} \hat{g}(\gamma) .
$$

Hence, $\|W f\|_{\dot{B}_{2,1}^{1 / 2}}=\frac{1}{2 \pi}\|\hat{g}\|_{\dot{B}_{2,1}^{1 / 2}}$.

As a consequence of these remarks, it follows that $W$ maps $\mathcal{B}_{2,1}(\mathbf{R})$ continuously into $\dot{B}_{2,1}^{1 / 2}(\mathbf{R})$ and is invertible on its range. The same argument as in Theorem 4(b) shows that $W$ is surjective and completes the proof.

\section{EXTENSIONS TO HIGHER DIMENSIONS}

The extension of the Wiener-Plancherel formula to higher dimensions is nontrivial. Several extensions, based on differing geometries for $\mathbf{R}^{d}$, have recently been derived $[\mathrm{BBE}]$, [Bene], [Benk]. For example, the "rectangular" Wiener transform of $[\mathrm{BBE}]$ is

$$
W f(\gamma)=\int_{\mathbf{R}^{d}} f(t) \mathcal{E}(t, \gamma) d t, \quad \text { where } \mathcal{E}(t, \gamma)=\prod_{i=1}^{d} \frac{e^{-2 \pi i t_{j} \gamma_{j}}-\chi_{[-1,1]}\left(t_{j}\right)}{-2 \pi i t_{j}} .
$$

The Wiener-Plancherel formula is then

$$
\lim _{T \rightarrow \infty} \frac{1}{\left|R_{T}\right|} \int_{R_{T}}|f(t)|^{2} d t=\lim _{\lambda \rightarrow 0} \frac{2^{d}}{\lambda_{1} \cdots \lambda_{d}} \int_{\mathbf{R}^{d}}\left|\Delta_{\lambda} W f(\gamma)\right|^{2} d \gamma
$$

where $R_{T}=[-T, T]^{d}, \Delta_{\lambda}$ is the rectangular symmetric difference operator

$$
\Delta_{\lambda} F(\gamma)=\frac{1}{2^{d}} \sum_{\omega \in\{-1,1\}^{d}}(-1)^{|\omega|} F(\gamma+\lambda \omega),
$$

and the limits are taken according to a particular natural convergence criterion. 
Our results all extend to the setting of [BBE]. The Besicovitch space $\mathcal{B}_{p, q}\left(\mathbf{R}^{d}\right)$ is defined by the norm

$$
\|f\|_{\mathcal{B}_{p, q}}=\left(\int_{\mathbf{R}_{+}^{d}}\left(\frac{1}{\left|R_{T}\right|} \int_{R_{T}}|f(t)|^{p} d t\right)^{q / p} \frac{d T}{T_{1} \cdots T_{d}}\right)^{1 / q}
$$

and a "rectangular" Besov space norm is defined by

$$
\|F\|_{\dot{B}_{p, q}^{s}}=\left(\int_{\mathbf{R}_{+}^{d}}\left(\frac{2^{d}}{\left(\lambda_{1} \cdots \lambda_{d}\right)^{s}}\left(\int_{\mathbf{R}^{d}}\left|\Delta_{\lambda} F(\gamma)\right|^{p} d \gamma\right)^{1 / p}\right)^{q} \frac{d \lambda}{\lambda_{1} \cdots \lambda_{d}}\right)^{1 / q} .
$$

Easy extensions of our results show that this Wiener transform is a topological isomorphism of $\mathcal{B}_{2, q}\left(\mathbf{R}^{d}\right)$ onto $\dot{B}_{2, q}^{1 / 2}\left(\mathbf{R}^{d}\right)$ for $1<q \leq \infty$, etc. We expect that similar results should also hold true using the spherical Wiener transform of [Bene] or [Benk].

\section{ACKNOWLEDGMENT}

We thank John Benedetto for discussions on the Wiener transform and the Wiener-Plancherel formula.

\section{REFERENCES}

[BBE] J. Benedetto, G. Benke, and W. Evans, An n-dimensional Wiener-Plancherel formula, Adv. in Appl. Math. 10 (1989), 457-487. MR 91h:42014

[Bene] J. Benedetto, The spherical Wiener-Plancherel formula and spectral estimation, SIAM J. Math. Anal. 22 (1991), 1110-1130. MR 92h:42028

[Benk] G. Benke, A spherical Wiener-Plancherel formula, J. Math. Anal. Appl. 171 (1992), 418435. MR 94a:43016

[Beur] A. Beurling, Construction and analysis of some convolution algebras, Ann. Inst. Fourier (Grenoble) 14 (1964), 1-32. MR 32:321

[CL] Y.-Z. Chen and K.-S. Lau, Wiener transformation of functions with bounded averages, Proc. Amer. Math. Soc. 108 (1990), 411-421. MR 90g:42018

[F] H. G. Feichtinger, An elementary approach to Wiener's third Tauberian theorem, Sympos. Math. 29 (1988), 267-301. MR 89i:42023

[HW] P. Hartman and A. Wintner, The $\left(L^{2}\right)$-space of relative measure, Proc. Nat. Acad. Sci. 33 (1947), 128-132.

[M] J. Marcinkiewicz, Une remarque sur les espaces de M. Besikowitch, C. R. Acad. Sci. Paris 208 (1939), 157-159.

[T] H. Triebel, Theory of Function Spaces II, Birkhäuser, Boston, 1992. MR 93f:46029

[W] N. Wiener, Generalized harmonic analysis, Acta Math. 55 (1930), 117-258.

School of Mathematics, Georgia Institute of Technology, Atlanta, Georgia 30332 0160

E-mail address: heil@math.gatech.edu 